\title{
Transcriptome and metabolome analysis of Citrus fruit to elucidate puffing disorder
}

\author{
Ana M. Ibáñez ${ }^{a, 1}$, Federico Martinelli ${ }^{a, b, c, 1}$, Russell L. Reagan ${ }^{a}$, Sandra L. Uratsu ${ }^{a}$, \\ Anna Vo ${ }^{a}$, Mario A. Tinoco ${ }^{a}$, My L. Phu ${ }^{a}$, Ying Chen ${ }^{d}$, David M. Rocke ${ }^{d}$, \\ Abhaya M. Dandekar ${ }^{\mathrm{a}, *}$ \\ a Department of Plant Sciences, University of California, One Shields Avenue, Mail Stop 4, Davis, CA 95616, USA \\ ${ }^{\mathrm{b}}$ Department of Agricultural and Forest Sciences, Università degli Studi di Palermo, Viale delle Scienze, 90128 Palermo, Italy \\ c I.E.M.E.S.T. Istituto Euro Mediterraneo di Scienza e Tecnologia, Via Emerico Amari, 123, 90139 Palermo, Italy \\ ${ }^{\mathrm{d}}$ Division of Biostatistics, Med Sci 1C, Room 146, University of California, Davis, One Shields Avenue, Davis, CA 95616, USA
}

\section{A R T I C L E I N F O}

\section{Article history:}

Received 26 August 2013

Received in revised form 31 October 2013

Accepted 6 December 2013

Available online 14 December 2013

\section{Keywords:}

Albedo breakdown

Citrus

Fruit disorder

Metabolomics

Puffing

Transcriptomics

\begin{abstract}
A B S T R A C T
A systems-level analysis reveals details of molecular mechanisms underlying puffing disorder in Citrus fruit. Flavedo, albedo and juice sac tissues of normal fruits and fruits displaying symptoms of puffing disorder were studied using metabolomics at three developmental stages. Microarrays were used to compare normal and puffed fruits for each of the three tissues. A protein-protein interaction network inferred from previous work on Arabidopsis identified hub proteins whose transcripts show significant changes in expression. Glycolysis, the backbone of primary metabolism, appeared to be severely affected by the disorder, based on both transcriptomic and metabolomic results. Significantly less citric acid was observed consistently in puffed fruits. Gene set enrichment analysis suggested that glycolysis and carbohydrate metabolism were significantly altered in puffed samples in both albedo and flavedo. Expression of invertases and genes for sucrose export, amylose-starch and starch-maltose conversion was higher in puffed fruits. These changes may significantly alter source-sink communications. Genes associated with gibberellin and cytokinin signaling were downregulated in symptomatic albedo tissues, suggesting that these hormones play key roles in the disorder. Findings may be applied toward the development of early diagnostic methods based on host response genes and metabolites (i.e. citric acid), and toward therapeutics based on hormones.
\end{abstract}

(c) 2013 Elsevier Ireland Ltd. All rights reserved.

\section{Introduction}

Citrus is a source of complex carotenoids with anti-oxidant properties important to human health. The citrus fruit consists of two distinct regions: the pericarp (peel) and endocarp (juice sacs). The pericarp consists of two tissue layers: the outer flavedo and the albedo. Flavedo contains cellulose and other components such as essential oils, paraffin waxes, steroids, triterpenoids, fatty acids,

Abbreviations: ABA, abscisic acid; APG6, albino pale green 6; BA, brassinosteroid; $C D C 2$, cell division control 2; CHO, carbohydrate; FDR, false discovery rate; FPS2, farnesyl diphosphate synthase 2; GABA, $\gamma$-aminobutyric acid; GA, gibberelic acid; GAs, gibberelins; GASA, gibberellin-responsive protein 4; GC-MS, gas chromatography-mass spectrometry; GSEA, gene set enrichment analysis; HB, homeobox; HSP, heat shock proteins; IAA, indole-3-acetic acid; NADH, nicotinamide adenine dinucleotide (reduced form); PEP, phosphoenolpyruvate; PPDK, pyruvate orthophosphate dikinase; PPI, protein-protein interactions; SA, salicylic acid; TCA, tricarboxylic acid; TF, transcription factor.

* Corresponding author. Tel.: +1 5307527784; fax: +1 5307528502.

E-mail address: amdandekar@ucdavis.edu (A.M. Dandekar).

1 Both the authors contributed equally to this work. pigments, the bitter compound limonene, and enzymes. It is rich in oil glands and composed of several cell layers that become progressively thicker toward the inside. It is covered with wax and contains few stomata. In ripe fruit, flavedo cells contain chromoplasts with carotenoids (xanthophyll) inside, synthesized from chlorophyll, that are responsible for the fruit's color change during ripening. The albedo, or mesocarp, is the fleshy middle layer of the pericarp, between the exocarp and the endocarp. It is generally colorless and spongy, and changes its character and thickness throughout fruit development. Its properties determine ease of peeling. Albedo tissue is very physiologically active and is a place where disorder and disease symptoms commonly appear [1,2]. Many physiological disorders occur preharvest and are believed to result from either mineral deficiency (creasing, boron and, copper deficiency) or weather (water spots, zebra skin, sunburn, wind scar, and freezing injury) [3].

Several candidate genes with altered expression during peel development were reported in a broad survey of gene expression during rind development in "Washington Navel" orange using a customized microarray platform [4]. Normal development of albedo tissue is of particular interest because consumers avoid 
fruits that are visually abnormal. Processed and fresh citrus production is seriously threatened by diseases and physiological disorders, threatening the livelihood of individual growers and depriving consumers of locally produced fresh fruit. Peel disorders diminish fruit quality and thus the profits of growers and producers. One of the best-known physiological disorders is "puffing" disorder, characterized by albedo breakdown and separation between peel and pulp. This leads to disintegration of the albedo tissue, causing the formation of air spaces and albedo with weaker mechanical resistance during peeling in mature fruits. Symptoms increase if the peel continues growing after the pulp has completed its development. The cause of this fruit disorder is currently unknown, although it seems to be associated with water exchange regulation through peel tissues or mineral nutrition [5]. Before the present study, no investigations were performed to elucidate molecular causes of the disorder.

This study examined the transcriptome and metabolome of citrus fruits to understand the disorder at the molecular level. Transcriptomics, the quantification of transcripts in a cell, includes the use of next-generation techniques [6-9] and microarrays [10-12]. Metabolomics may be a good complement to transcriptomics and proteomics [13-16]. The metabolome is more directly related to phenotype than transcripts or proteins because metabolic fluxes are governed not only by gene expression, but also by post-transcriptional and post-translational events. Gas chromatography-mass spectrometry (GC-MS) is typically used for non-targeted metabolite profiling of volatile, thermally stable polar and non-polar metabolites [17].

A microarray analysis was performed on three different fruit tissues (flavedo, albedo, and juice sacs) to compare normal and puffed samples, followed by metabolomic profiling of peel and juice sacs. The two primary objectives were: (1) to identify genes and metabolites with variability corresponding to symptoms of puffing disorder; and (2) to identify pathways that describe network regulation responsible for the disorder. Fruit peel tissues can serve as a biological sensor of abiotic and biotic stresses. They contain key biomarkers for early diagnosis and possible therapies for diseases and disorders. The identification of genes, metabolites and regulatory interactions from this study provides knowledge essential for the development of early diagnostic methods and novel therapeutic strategies based on small molecule applications.

\section{Materials and methods}

\subsection{Plant material and experimental design}

Symptomatic and healthy control "Navel" orange fruits were harvested at three different developmental stages (immature, intermediate, and mature) from an orchard located in San Diego County, California, USA. Symptoms of puffing become apparent at the mature "Navel" orange stage. Thus immature and intermediate fruits that were harvested from trees that showed the typical puffing symptoms in mature fruit during the previous season were considered to be symptomatic. The study included four immature, four intermediate, and four mature symptomatic fruits, each comprising one biological replicate. Two healthy fruits comprised two biological replicates of control samples. Mature, healthy fruits of five different citrus cultivars (mandarin [Citrus reticulata], "Valencia" and "Navel" orange [Citrus sinensis], lemon [Citrus $\times$ limon], grapefruit [Citrus $\times$ paradisi]) were purchased from a food market located in Davis, CA, USA. Three fruits comprised three biological replicates from each of the five different healthy citrus cultivars. At the time of collection of each fruit sample, a $1 \mathrm{~cm}$-thick equatorial disk and four sections (N, S, E, and W) were cut per fruit. Flavedo, albedo, and juice sac from each section was dissected, ground in liquid nitrogen and stored at $-80^{\circ} \mathrm{C}$ until analyzed. Metabolomic analysis was conducted at different developmental stages (immature, intermediate, and mature) for each of the three tissues, and for both symptomatic and control fruits.

\subsection{Metabolomic analysis}

Analysis of metabolites was conducted at the Metabolomics Core facility at the UC Davis Genome Center. "Navel" orange samples were collected at three developmental stages (immature, intermediate, and mature), for each of the three tissues, for both symptomatic and control samples. Metabolites from 20 to $50 \mathrm{mg}$ frozen, ground samples were extracted with $1 \mathrm{~mL}$ pre-chilled solvent mixture $\left(\mathrm{dH}_{2} \mathrm{O}:\right.$ MetOH: $\mathrm{CHCl}_{3}$ 1:2.5:1), vortexed for about $10 \mathrm{~s}$ and shaken for $6 \mathrm{~min}$ at $4{ }^{\circ} \mathrm{C}$. Samples were then centrifuged for $2 \mathrm{~min}$ at $14,000 \mathrm{rpm}$ and supernatant was removed. $500 \mu \mathrm{L}$ aliquots of supernatant were concentrated to complete dryness in a speed vacuum concentrator. Dry samples were stored at $-20^{\circ} \mathrm{C}$ until analyzed. Reconstituted metabolite samples were analyzed with a Pegasus III TOF GC-mass spectrometer that can profile 400 compounds. Relative concentrations were determined by peak area $\left(\mathrm{mm}^{2}\right)$. All peak detections were manually checked for false positive and false negative assignments. These mass spectra were then compared to known and commercially available mass spectral libraries. Statistical analysis was performed using pairwise comparison to determine significant differences. Target GC-MS metabolite analysis was performed for sucrose, fructose, glucose, citric, and malic acid.

\subsection{RNA extraction}

For transcript analysis, total RNA was extracted from $2 \mathrm{~g}$ of pooled $(\mathrm{N}+\mathrm{S}+\mathrm{E}+\mathrm{W})$ sections of each fruit and tissue (albedo, flavedo, or juice sac). Total RNA was extracted using a two-day modified hot borate procedure [18], followed by RNA purification using Qiagen RNeasy cleanup kit (Qiagen; Valencia, CA). RNA concentration and purity were assessed by UV spectrophotometry. RNA was stored at $-80^{\circ} \mathrm{C}$ until analyzed.

\subsection{Microarray analysis}

RNA labeling was performed according to instructions in the GeneChip One Cycle Target Labeling Kit (Affymetrix, Inc., Santa Clara, CA, USA). Citrus microarrays were purchased from Affymetrix. Each microarray contains over 30,171 probe sets corresponding to 33,879 citrus gene transcripts. Fragmentation, hybridization, scanning, and image data processing were performed according to Affymetrix protocols at the Microarray Core Facility in the Medical Microbiology and Immunology Department, University of California, Davis (USA).

\subsection{Statistical analysis of microarray data}

The raw probe intensities obtained after scanning individual citrus chips were processed using the RMA method to normalize and scale the data [19]. To determine which genes were differentially expressed among different groups, one-way ANOVA was used to obtain $p$-values for each gene. All $p$-values were BH-adjusted for multiple hypotheses [20]. Genes with adjusted $p$-values $<0.05$ were considered differentially expressed (up or downregulated). $R$ package LMGene [21] was used to perform a one-way ANOVA and adjust for multiple hypotheses. $\mathrm{R}$ package limma was used to make pairwise comparisons between groups and identify citrus fruit- and tissue-specific genes. 


\section{6. qPCR analysis}

For each target gene, PCR primers and a TaqMan probe were designed using Primer Express software (Applied Biosystems, Foster City, CA, Table S1). DNase treatment and cDNA synthesis were completed following a combined protocol based on the Quantitect Reverse Transcription Kit (Qiagen Inc., Germany). A standard curve determined the linearity of amplicon quantity vs initial cDNA quantity for each gene. Five $\mu \mathrm{L}$ cDNA at $5 \mathrm{ng} / \mu \mathrm{L}$ was diluted to a $12-\mu \mathrm{L}$ final volume using TaqMan Universal PCR Master Mix. Amplifications were performed using standard amplification conditions: $2 \mathrm{~min}$ at $50^{\circ} \mathrm{C}, 10 \mathrm{~min}$ at $95^{\circ} \mathrm{C}$, and 40 cycles of $15 \mathrm{~s}$ at $95^{\circ} \mathrm{C}$ and $60 \mathrm{~s}$ at $60^{\circ} \mathrm{C}$. Fluorescent signals were collected during the annealing temperature and $\triangle \mathrm{CT}$ was calculated. $C$. sinensis elongation factor 1- $\alpha$ (accession number: AY498567) was used as an endogenous reference.

Supplementary data related to this article can be found in the online version, at http://dx.doi.org/10.1016/j.plantsci.2013.12.003.

\subsection{Bioinformatic analysis}

Annotations of some citrus sequences were supplied by Affymetrix. Additional annotations were found by BLAST comparisons with the NCBI non-redundant protein and TAIR databases. Genes passing criteria for differential expression (adjusted $p$-value $<0.05$ ) were visualized and functionally analyzed using MapMan [22]. PathExpress software [23] using default settings was used to interpret pathway regulation associated with different fruit types and conditions. Blast2GO software was used to perform Fischer's exact tests to identify GO-terms significantly enriched in puffed fruit samples [24]. For protein-protein interaction (PPI) analysis, an interaction network for Citrus was inferred based on a PPI network for Arabidopsis [25]. Interactive proteins encoded by differentially regulated genes were identified and visualized using Graphviz software.

\section{Results and discussion}

\subsection{Metabolomic changes associated with puffing disorder}

Following a systems biology approach, an untargeted metabolomic analysis was conducted to identify which metabolites varied in abundance between puffed and control fruits in flavedo, albedo, and juice sacs at three fruit developmental stages. Puffing disorder is typically observed only in mature fruits. To identify the causes of the disorder, however, it is useful to analyze fruits before symptoms appear. We analyzed the immature fruit of trees that had displayed the disorder in most fruits during the previous season. These immature fruits were assumed to have a high probability of developing the disorder. The observation that a metabolite concentration changes at both the pre-symptomatic immature and symptomatic mature stages may corroborate the hypothesis that these metabolites are linked with the development of the disorder. An alternative hypothesis would assert that a transient fluctuation of metabolites occurred at the time of sampling.

Of approximately 400 metabolites analyzed, 42 were significantly changed between healthy and puffed fruits at the same developmental stage, among which 15 were identified (Fig. 1). No significant changes were observed in flavedo tissue. More differences were observed in albedo than in mature juice sacs. Pipecolic acid, alanine, citric acid, $\gamma$-aminobutyric acid (GABA), and levanbiose decreased in symptomatic fruits while galactinol, raffinose, glucose-1-phosphate, inulotriose, oxoproline, ribitol, and vanillic acid increased. Interestingly, citric acid, a typical compound of citrus fruits, decreased in symptomatic albedo at all three developmental stages. Target metabolite analysis using GC-MS confirmed the reduced concentration of citric acid in puffing-affected fruits. This important finding opens the possibility of using citric acid as an early biomarker for puffing disorder: its concentration can be measured quickly by laboratory assay or directly in orchards using tools currently under development. Citric acid is a key indicator of citrus fruit quality that accumulates in immature fruit, then decreases during ripening. The isomerization of citrate to isocitrate is catalyzed by aconitase. Juice sacs contained more galactinol and tryptophan in samples of immature and mature puffed fruits, and more raffinose at all three developmental stages. Succinic acid was more abundant in mature disordered fruits only. Glucose-1-phosphate was more abundant in albedo of puffed fruits, consistent with downregulation of phosphoglucomutase transcripts. Gene set and pathway enrichment analysis showed that amino acid and derivative metabolic processes were significantly affected in albedo (Table 1), consistent with variations in abundance of alanine and oxoproline. Target GC-MS analysis showed no significant differences in sucrose, fructose, and glucose. Malic acid was also unchanged between normal and puffed fruits in all three tissues.

\subsection{Microarray analysis of Citrus fruit}

Two microarray analyses were performed. The first one compared healthy "Navel" orange fruits and those with puffing disorder in each of three tissues (GEO: GSE49957). The second examined differences and similarities in the transcriptomes of different healthy Citrus fruit types: mandarin orange, lemon, grapefruit, "Valencia" orange, and "Navel" orange (GEO: GSE50384). The latter dataset provides a basic knowledgebase of variability among five consumer varieties of Citrus fruit for further studies on fruit development, ripening, and quality improvement. Both data analyses were integrated to identify which disorder-related genes also vary among different Citrus fruits.

An unsupervised clustering of differentially expressed genes was carried out. This analysis encompasses genes from microarray studies of puffing disorder in flavedo, albedo, and juice sac tissues, as well as those found in the 10 pairwise comparisons between the five types of fruit in the three tissues. This is presented as a Venn diagram (Fig. S1). Pathway enrichment analysis showed that phenylpropanoid metabolism was affected by gene regulation among Citrus fruit types in all three tissues (Table S2). Fatty acid biosynthesis, the pentose phosphate pathway, tyrosine metabolism, limonene, and pinene degradation were other pathways that varied in flavedo of different Citrus fruits. In the albedo of different fruits, other pathways were affected: flavonoid, anthocyanin, and carotenoid biosynthesis and ascorbate and aldarate metabolism. In juice sacs, most transcriptional variation occurred in important primary metabolic pathways such as glycolysis, carbohydrate metabolism (fructose, mannose, starch, and sucrose metabolism), flourene degradation, biotin, glutathione metabolism, and 3-chloroacrylic acid degradation. Highly differentially expressed genes in each pairwise fruit comparison are of interest for understanding differences among transcriptomes of citrus types (Table S3). The transcriptome profiles of "Valencia" and "Navel" oranges were similar: only 21 differentially expressed genes were identified in this pairwise comparison $\left(p<10^{-4}\right)$. Two other pairwise comparisons with few transcriptomic differences were seen between mandarin and "Navel" orange, and between "Valencia" orange and grapefruit. Determining which genes are commonly regulated among different Citrus species provides a baseline control for the study of puffing disorder within a given Citrus fruit type. 


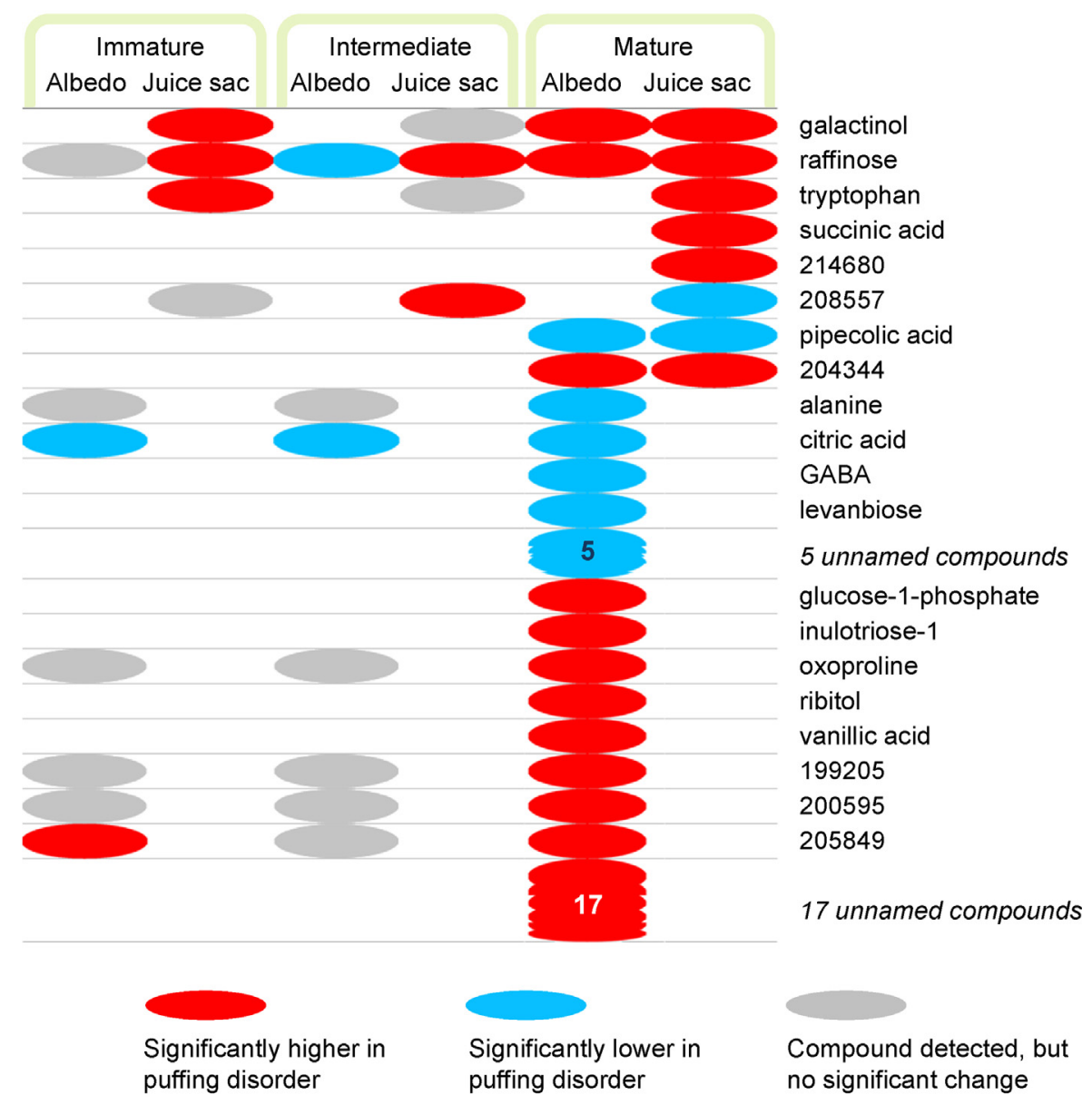

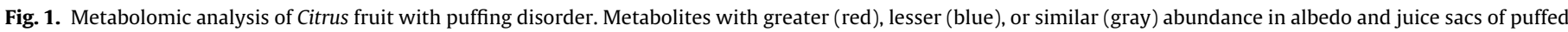

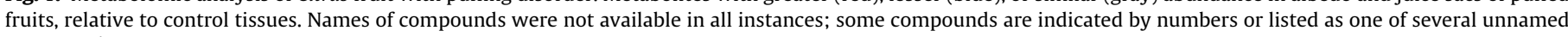
compounds.

Supplementary data related to this article can be found in the online version, at http://dx.doi.org/10.1016/j.plantsci.2013.12.003.

\subsection{Gene enrichment and functional analysis of puffing disorder}

The major objective of this work was a comprehensive analysis of puffing disorder. An analysis of the peel transcriptome adds insight into the causes of peel disorders, since peel acts as a biosensor of stress. Changes in the transcriptome of juice sacs are expected to correlate with changes in juice quality. The functions of genes highlighted by the transcriptome data were dissected using gene set and pathway analysis. The analysis identified genes that may regulate disorder-associated pathways. Analysis with Blast2GO, Pathexpress, and MapMan software produced similar findings.

Table 1

Gene sets enriched in tissues of puffed fruits, compared to control samples, found using Fisher's exact test.

\begin{tabular}{|c|c|c|c|}
\hline Tissue and regulation & GO term & Name & FDR \\
\hline \multirow[t]{6}{*}{ Upregulated flavedo } & GO:0016020 & Membrane & $2.87 \mathrm{E}-09$ \\
\hline & GO:0005215 & Transporter activity & $2.59 \mathrm{E}-06$ \\
\hline & GO:0006811 & Ion transport & $9.14 \mathrm{E}-04$ \\
\hline & GO:0005794 & Golgi apparatus & 0.01053 \\
\hline & GO:0005975 & Carbohydrate metabolic process & 0.023832 \\
\hline & GO:0016787 & Hydrolase activity & 0.025858 \\
\hline Downregulated flavedo & None & None & None \\
\hline \multirow[t]{6}{*}{ Upregulated albedo } & GO:0016020 & Membrane & $1.35 \mathrm{E}-08$ \\
\hline & GO:0006810 & Transport & $1.51 \mathrm{E}-07$ \\
\hline & GO:0005215 & Transporter activity & $9.11 \mathrm{E}-07$ \\
\hline & GO:0005975 & Carbohydrate metabolic process & 0.019001 \\
\hline & GO:0005783 & Endoplasmic reticulum & 0.020346 \\
\hline & GO:0004871 & Signal transducer activity & 0.047297 \\
\hline \multirow[t]{4}{*}{ Downregulated albedo } & GO:0040029 & Regulation of gene expression, epigenetic & 0.002355 \\
\hline & GO:0009536 & Plastid & 0.011954 \\
\hline & GO:0006519 & Amino acid and derivative metabolic process & 0.026269 \\
\hline & GO:0004518 & Nuclease activity & 0.035601 \\
\hline
\end{tabular}

Over-represented GO terms among differentially expressed genes in albedo and flavedo tissues affected by puffing disorder are shown. Annotated genes with GO terms from the Affymetrix GeneChip were used as the reference gene set, with FDR $<0.05$. 
Table 2

Differentially regulated pathways and $p$-values in three citrus fruit tissues associated with puffing disorder.

\begin{tabular}{|c|c|c|c|}
\hline \multirow[t]{2}{*}{ Pathway } & \multicolumn{3}{|c|}{ Fruit tissue } \\
\hline & Flavedo & Albedo & Juice sacs \\
\hline Limonene and pinene degradation & 0.004 & 0.046 & 0.016 \\
\hline Lipopolysaccharide biosynthesis & 0.002 & 0.004 & n.s. \\
\hline Anthocyanin biosynthesis & 0.02 & 0.038 & n.s. \\
\hline Biosynthesis of unsaturated fatty acids & 0.006 & 0.013 & n.s. \\
\hline Starch and sucrose metabolism & 0.009 & n.s. & n.s. \\
\hline Propanoate metabolism & 0.014 & n.s. & n.s. \\
\hline 2,4-Dichlorobenzoate degradation & 0.016 & n.s. & n.s. \\
\hline Fluorene degradation & 0.016 & n.s. & n.s. \\
\hline Ethylbenzene degradation & 0.016 & n.s. & n.s. \\
\hline 1- and 2-Methylnaphthalene degradation & 0.006 & n.s. & n.s. \\
\hline Naphthalene and anthracene degradation & 0.02 & n.s. & n.s. \\
\hline Insect hormone biosynthesis & 0.046 & n.s. & n.s. \\
\hline Bisphenol A degradation & 0.046 & n.s. & n.s. \\
\hline Benzoxazinone biosynthesis & 0.046 & n.s. & n.s. \\
\hline Pentose phosphate pathway & n.s. & 0.006 & n.s. \\
\hline Methionine metabolism & n.s. & 0.016 & n.s. \\
\hline Phenylpropanoid biosynthesis & n.s. & n.s. & 0.012 \\
\hline Selenoamino acid metabolism & n.s. & n.s. & 0.024 \\
\hline Aminoacyl-tRNA biosynthesis & n.s. & n.s. & 0.028 \\
\hline
\end{tabular}

Analysis was performed using PathExpress software. P-values $<0.05$ are indicated. "n.s." means not significant.

Gene set enrichment analysis (GSEA) using Blast2GO focused on the two peel tissues of specific interest in puffing disorder, flavedo and albedo (Table 1). For both peel tissues of puffed and normal fruits, there was significant upregulation of genes in general transport pathways (transporter activity, ion transport, and membrane) and carbohydrate metabolism. No gene ontology terms were significantly downregulated by puffing disorder in flavedo (FDR $<0.5$ ). In albedo, downregulated pathways were related to gene transcriptional regulation, amino acid, and nuclease activity.

Pathway enrichment analysis showed that limonene and pinene degradation pathways were significantly affected by the disease in all three tissues (Table 2). In flavedo, 15 pathways were significantly affected by puffing disorder $(p<0.05)$. Other volatile pathways that appear to be highly altered in puffing disorder include anthocyanin and unsaturated fatty acid biosynthesis and lipopolysaccharide, starch, and sucrose metabolism. In albedo, the pentose phosphate pathway and methionine biosynthesis were affected.

\subsection{Primary metabolism}

Expression of genes responsible for the backbone pathways of primary metabolism such as glycolysis, the tricarboxylic acid (TCA) cycle, sucrose, starch, and raffinose metabolism differed between puffed and control fruits, as shown in the metabolism overview (Fig. 2) provided by MapMan [26]. Genes encoding the glycolytic enzymes phosphoglucomutase, glucose-6-phosphate isomerase, and phosphofructokinase were downregulated (Fig. 3). In the energy payoff phase of the glycolytic pathway, expression of genes in pyruvate and phosphoenolpyruvate $(P E P)$ metabolism increased in puffed fruits, including pyruvate orthophosphate dikinase (PPDK) (upregulated in flavedo) and phophoenolpyruvate carboxylase (upregulated in both flavedo and albedo). Initial TCA cycle pathway genes encoding aconitate hydratase and isocitrate dehydrogenase were downregulated. The finding in metabolomics analysis that malic acid was unaltered contrasts with the observed upregulation of malate synthase. This could be due to post-transcriptional regulation of this gene.

Expression of genes involved in mitochondrial redox reactions such as electron transport and ATP synthesis (NADH-DH, cytochrome c oxidase, and $\mathrm{g}$ subunit family protein) differed between puffed and control fruits. Invertase was upregulated in both flavedo and albedo (Fig. 4A). Different starch synthesis genes showed contrasting results: ADP glucose pyrophosphorylase and starch synthase transcripts were downregulated, while a gene encoding a starch branching enzyme was upregulated in puffed fruits, relative to controls. The expression patterns of specific invertase genes encoding isoenzymes with different subcellular localization are unclear. Variation among these isoforms could account for unchanged amounts of sucrose, glucose, and fructose in metabolomics results. Starch and sucrose metabolism pathways are also affected by biotic stresses such as Huanglongbing disease [8,26-28] and citrus bacterial canker disease [29] in Citrus (Table S4).

Supplementary data related to this article can be found in the online version, at http://dx.doi.org/10.1016/j.plantsci.2013.12.003.

Raffinose metabolism is directly linked to glycolysis and sucrose and starch metabolism. Transcripts encoding galactinol synthase and $\alpha$-galactosidase 1 were more abundant in puffed compared to control fruits, while those encoding glucose-6-phosphate isomerase and phosphoglucomutase were less abundant (Fig. 4B). Genes encoding different isoforms of myoinositol phosphatase, sugar epimerase, and carbohydrate kinase showed opposite regulation trends.

Regulation of glycolysis would suggest that changes in the abundance of adenosine triphosphate (ATP) might occur in puffing disorder. The reaction catalyzed by phosphofructokinase is the most important regulatory step of glycolysis, and the expression of its encoding gene was downregulated in puffed peel tissues. Interestingly, while genes involved in the energy investment pathway steps were expressed at lower levels in puffed samples, genes associated with reactions involved in the energy payoff phase were expressed more highly. TCA cycle genes such as fumarase and succinyl-CoA ligase were upregulated. These findings are consistent with studies showing that fumarase was upregulated in fruit tissues affected by core breakdown disorder in pear [30]. Further experiments at the proteomic and enzymatic levels may be necessary to determine whether this perturbation in glycolysis associated with puffing disorder decreases production of ATP, the reduced form of nicotinamide adenine dinucleotide (NADH), or other high energy cofactors. A gene encoding L-lactate dehydrogenase, which converts pyruvate to lactate (fermentation), increased expression in both flavedo and albedo tissues of puffed fruits, relative to control fruits. Phosphoenolpyruvate carboxylase 1 transcripts were more abundant in symptomatic flavedo tissue than in controls (Fig. 5). The conversion of pyruvate to lactate is typically induced by hypoxic conditions. Conversion of phosphoenolpyruvate to 


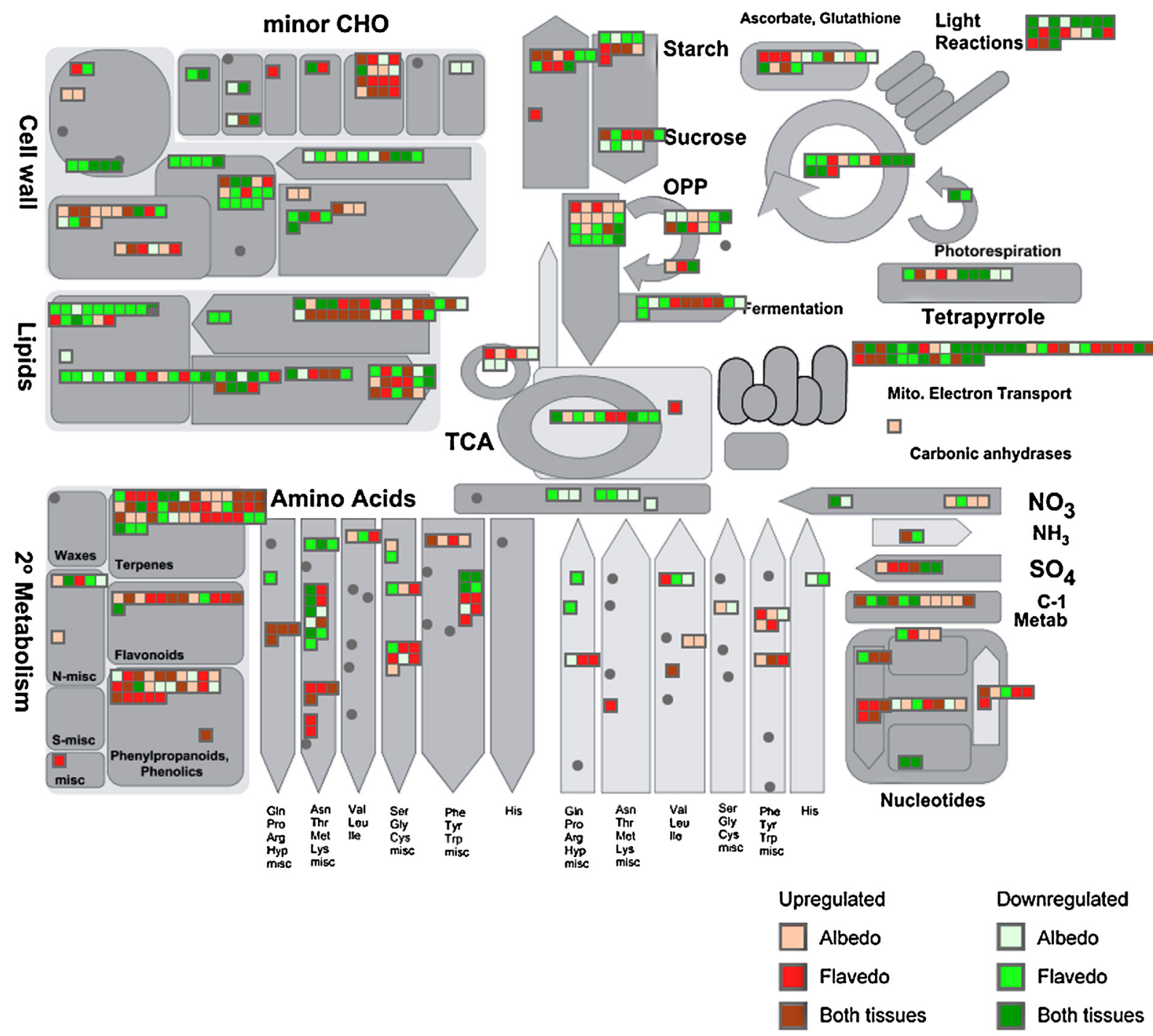

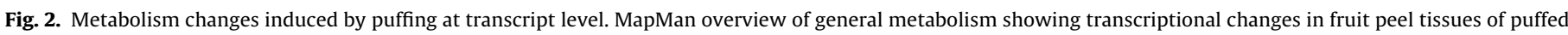
fruits, relative to control samples.

oxoalacetate is another key regulatory point of the glycolytic pathway. This step appears to be affected in puffing disorder. Oxidation of each molecule of glucose to pyruvate produces two ATP molecules. This reaction is essential to provide energy and for a wide range of other physiological functions [31].

It may be premature to conclude that alterations in the expression of genes involved in primary metabolism would diminish the energy available to peel cells for growth. Nevertheless, changes in glycolysis could affect other key pathways of primary metabolism such as starch, sucrose, and raffinose metabolism. Expression of sucrose-phosphate synthase (SPS) gene decreased, while that of invertase and sugar transporter increased in flavedo and albedo of puffed fruit, relative to control samples. This key transcriptional change could alter source-sink relations throughout the plant. Interestingly, SPS was upregulated during fruit development [32]. The same authors reported that the while invertase transcript abundance did not change, a gene encoding an invertase inhibitor was induced just prior to ripening. These genes play key roles in the responses of Citrus to Huanglongbing disease $[8,27,28]$. Starch metabolism was also affected: transcripts related to amylose and starch degradation pathways were more abundant in puffed fruits, compared to control fruits. Changes in transcripts of the raffinose pathway suggested that raffinose and galactinol concentrations should increase in tissues of puffed fruit, as observed at the metabolomic level (Fig. 1). These changes could modulate other important metabolic pathways, as sugars regulate expression of many genes. Some genes, such as those involved in photosynthesis, are repressed by sugars [33], while others are induced [34]. Sugars can affect gene expression indirectly as an energy source, directly as precursors for metabolic reactions, as osmotic regulators, or as signaling molecules. In most cases, sugar phosphorylation by hexokinase is required to initiate signal transduction [34]. Hexokinase has been proposed as a sugar-sensing component, with both catalytic and regulatory functions [32]. For example, crosstalk between sugar and gibberellins in developing petunia flowers modulates the expression of anthocyanin synthesis genes [35].

\subsection{Hormone-related pathways}

Responses to any environmental stress can be affected by hormone crosstalk. Most genes involved in gibberellin biosynthesis and signaling were downregulated in albedo tissue of puffed fruits (Fig. 4C). In flavedo, ent-kaurene synthase was downregulated. This gene encodes an enzyme in the gibberellin biosynthetic pathway catalyzing the second step in the 


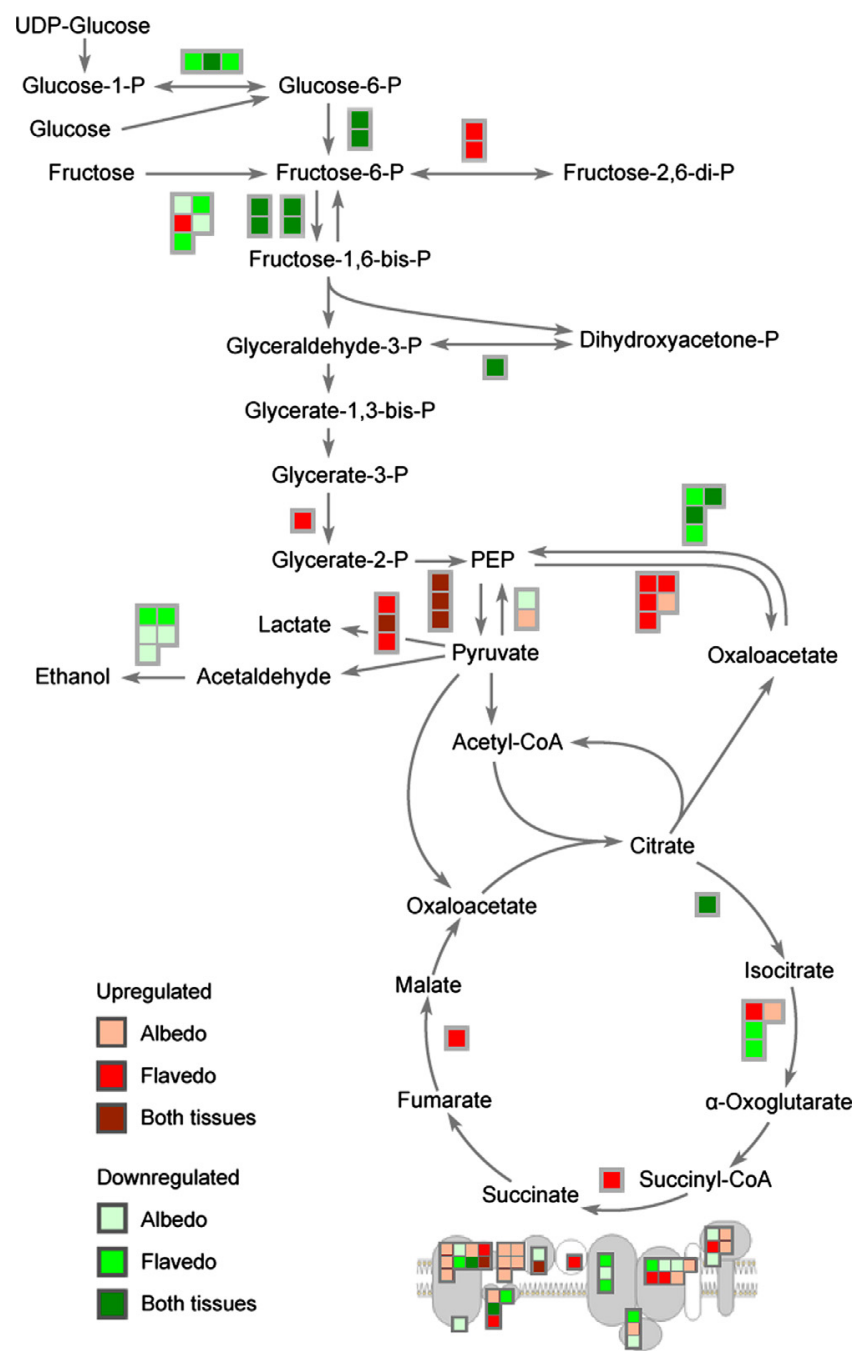

Fig. 3. Transcriptional changes in fruit primary metabolism induced by puffing disorder. Altered expression of genes in glycolysis and the TCA cycle in albedo and flavedo tissues during puffing disorder as visualized by MapMan.

cyclization of GGPP to ent-kaurene. In both peel tissues, ent-copalyl diphosphate synthase was downregulated (gibberellic acid (GA) requiring 1 ). In albedo, seven genes were downregulated: two oxidoreductases (2-OG-Fe(II)), GA insensitive, two belonging to a gibberellin-regulated family, and two GASAs. Genes involved in other hormone pathways such as a histidine kinase 1 (cytokinins) and an S-adenosylmethionine-dependent methyltransferase (salicylic acid) were significantly downregulated.

Expression of genes encoding components of ethylene, auxin, and brassinosteroid related pathways was significantly altered in puffed compared to control fruits. Twenty-two auxin-responsive genes were downregulated (six in albedo, four in flavedo, 12 in both peel tissues), but 10 were upregulated (eight in albedo and six in both peel tissues). Brassinosteroids play a key role in the regulation of fruit ripening. Some brassinosteroid-related transcripts (HYD1, DWF5, CYP90D1, and squalene epoxydase 1) were more abundant, as were others encoding DIM1, SQ3, and HYP6. Genes encoding ethylene-induced oxidoreductases and ACS1 and isoforms of ACO4 were induced by puffing disorder. Expression of most genes involved in ethylene signal transduction (ERF12, ERF13, $E R F 1$, and $A P 2-T F$ ) was diminished in puffed samples. Expression of EIN1 encoding an ethylene receptor also was lower. Unlike other genes involved in ethylene signaling, decreased expression of EIN1 could lead to an increase in sensitivity to the hormone.
Endogenous hormone balance plays a crucial role in regulating glycolysis, the TCA cycle, sucrose and starch metabolism, and source-sink communications [36,37]. Gibberellins (GAs) are essential endogenous regulators of plant growth and development [38]. Although the complexity of GA biosynthesis is not fully understood, various mechanisms that regulate GA concentrations in plants are known [39]. In albedo of puffed fruits, transcript abundance of genes involved in GA metabolism and signal transduction was lower than in controls (Fig. 4C). In addition, downregulation of gibberellin-responsive protein 4(GASA4) in both albedo and flavedo was validated by qRT-PCR (Fig. 5). The upregulation of some GRAS transcription factors is another indicator that gibberellin regulation is altered in puffing disorder. This family of transcription factors includes DELLA proteins, well-known negative regulators of gibberellin response [40]. These data concur with reported effects of $\mathrm{GA}_{3}$ applications before fruit color break, which decrease the occurrence of puffing in some Citrus fruits [1,41-43].

Gibberellins affect other physiological disorders in fruit, such as watercore development in pear [44,45]. Compared to untreated fruits, gibberellin-treated fruits are juicier, firmer, and suffer less rind damage during harvest, although they have undesiderable chlorophyll retention [46]. We speculate that downregulation of gibberellin genes is linked not only to puffing disorder but also to other disorders in Citrus and other fruit crops. Indeed, gibberellin regulation plays a key role in fruit physiology disorders. Gibberellins regulate glycolysis in wheat leaves [47]. In addition, triose-phosphate isomerase expression changes and $\mathrm{GA}_{3}$ application correlated positively during flower development [48]. The glycolytic enzyme fructose-bisphosphate aldolase was upregulated by $\mathrm{GA}_{3}$ application in rice roots [49]. Several studies have implicated a link between gibberellins and sugar metabolism. Soluble sugar content was regulated by application of $\mathrm{GA}_{3}$ in grapevine [50]. $\mathrm{GA}_{3}$ application increased sucrose-phosphate-synthase activity, triggering starch breakdown and sucrose synthesis [50]. These studies support a link between gibberellin downregulation and changes in glycolysis, the TCA cycle, and sucrose, starch and raffinose metabolism. The molecular basis for this link could be established in model plants for which mutants are available (Arabidopsis or tomato).

The upregulation of several genes involved in IAA metabolism (Fig. 4C) suggests they might help prevent the disorder, consistent with the beneficial effects of auxin and auxin analog applications before fruit color break [1]. Cytokinins are key regulators of plant development and growth, affecting tissue source or sink strength, carbon fixation and assimilation, partitioning of primary metabolites, and cell cycle activity [51]. A histidine kinase family gene was downregulated in puffing disorder. However, no data are available on the effect of experimental cytokinin applications on symptom severity. The involvement of each hormone type should be validated using different doses and environmental and physiological conditions.qRT-PCR experiments showed strong downregulation of cytokinin-independent 1 in albedo and to a lesser extent in flavedo of symptomatic fruits (Fig. 5). GASA4 and RanBP2 were strongly downregulated in symptomatic albedo and expressed at low levels in both control samples and flavedo of puffed fruits. These data were consistent with microarray results.

\subsection{Signaling pathways}

Over 200 genes involved in signaling pathways showed significant variation in transcript abundance in both albedo and flavedo tissues between puffed and control fruits (Fig. 6A). G-proteinrelated genes, including $S A R 1, R A B A 1, R A B C 1, R A B F 1, R A B 18 B$, and $R A B A 3$ were upregulated, along with some genes related to calcium regulation: calmodulin, ECT8, CAM7, ECT5, ACA1. Expression 
A

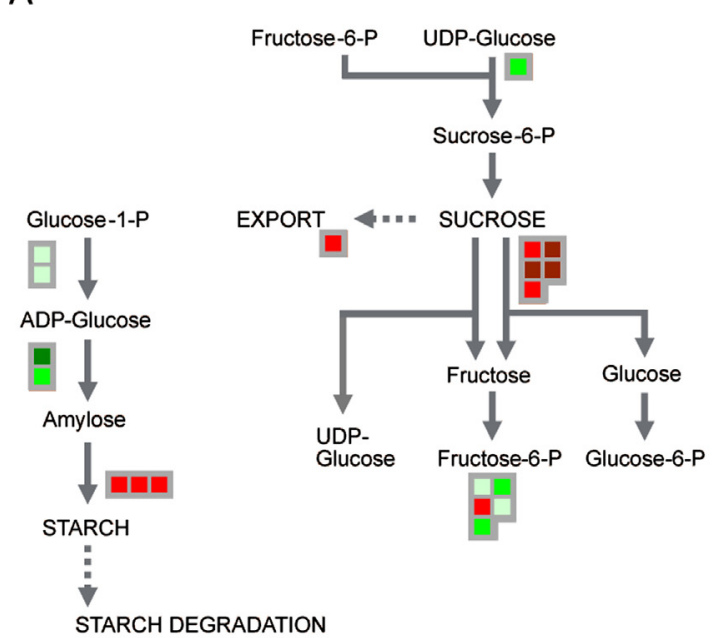

B

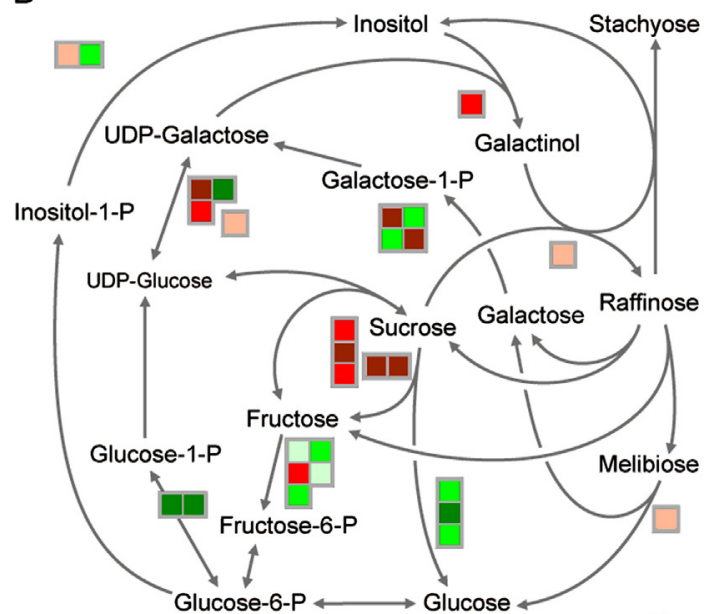

Upregulated

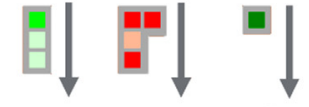

Glucose-1-P Maltose Glucose

EXPORT
C

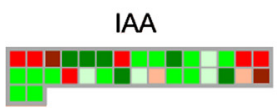

ABA

政

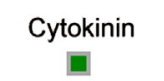

Jasmonate

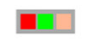

SA

口n
Albedo

Flavedo

Both tissues

Downregulated

Albedo

Flavedo

Both tissues

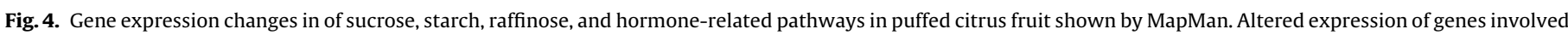
in sucrose and starch metabolism (A), raffinose metabolism (B), and hormone metabolism and signaling (C), in albedo and flavedo tissues during puffing disorder.

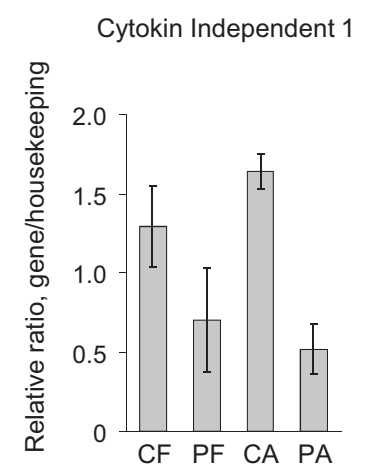

$$
\begin{array}{ll}
\text { L-Lactate Dehydrogenase } & \text { RanBP2 Zinc Finger } \\
& \text { Ran-binding } \\
& \text { Transcription Factor }
\end{array}
$$
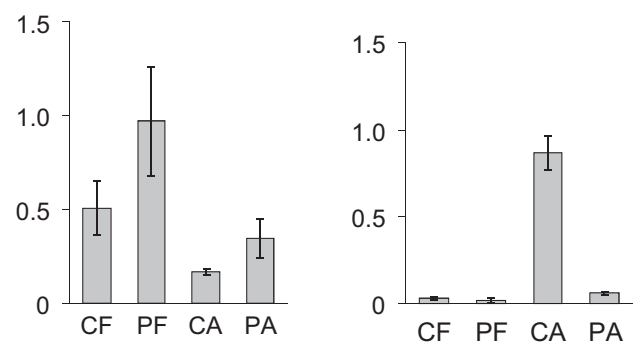

PEP Carboxylase

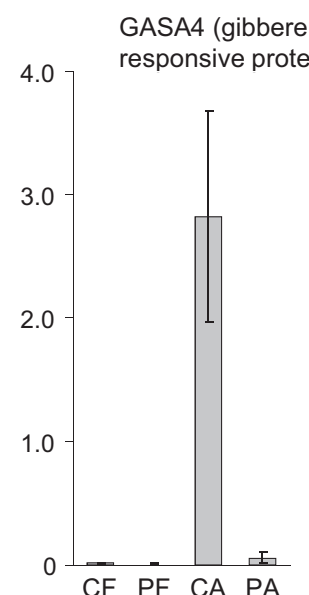

CF Control Flavedo

PF Puff Flavedo

CA Control Albedo

PA Puff Albedo

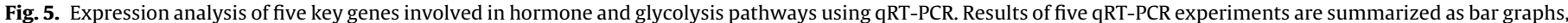
where vertical axes represent the relative ratio compared to the endogenous reference gene. Error bars represent standard deviations. 
A

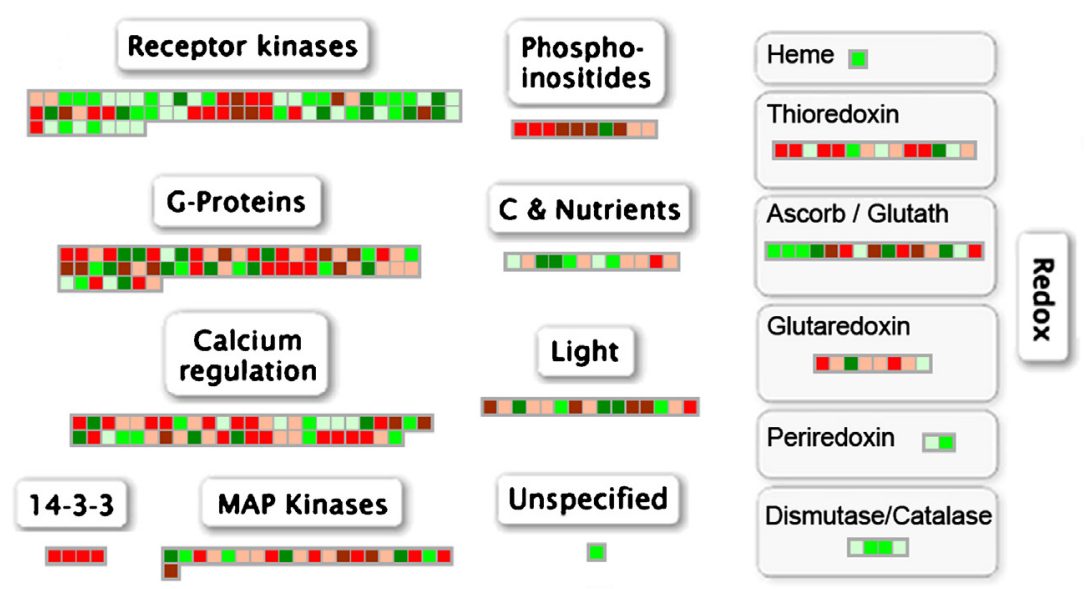

B
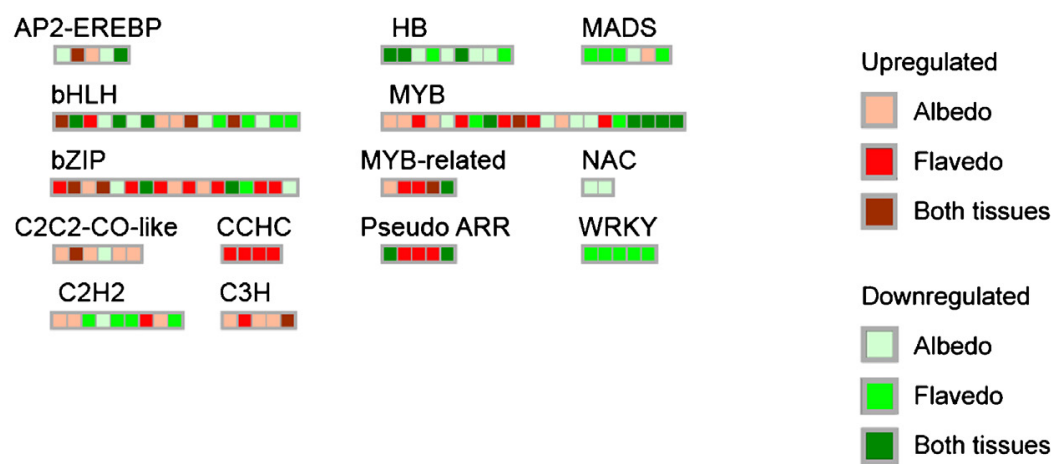

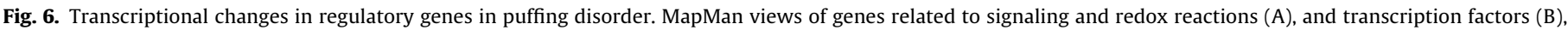
comparing fruit samples with puffing disorder and control samples.

of genes encoding 14-3-3 regulatory factors was higher (GRF2 and GRF8) in puffed samples.

\subsection{Redox pathways and transcription factors}

Redox reactions were altered, up or down, through regulation of several genes: thioredoxin, ascorbate/glutathione, glutaredoxin, periredoxin, and dismutase/catalase (Fig. 6A).

Transcription factors (TFs) also may play key roles in the gene regulatory networks causing puffing disorder (Table S5). NAC-related transcription factors were generally downregulated in albedo and flavedo of puffed fruits. Interestingly, NAC genes also are involved in the Citrus response to Huanglongbing [52]. In flavedo, repressed Aux/IAA transcription factors include IAA8, IAA16, IAA7, and PAP2. Aux/IAA, Homeobox (HB), WRKY57, WRKY68, WRKY32, WRKY75, and WRKY19 were repressed in peel tissues of symptomatic fruits (Fig. 6B). WRKY transcription factors have many regulatory roles in response to biotic and abiotic stresses [53]. WRKY75 was linked to susceptibility to virulent and avirulent isolates of Pseudomonas syringae [54]. WRKY25 and WRKY45 play important roles in responses to abiotic stresses such as thermotolerance and drought resistance in Arabidopsis [55]. The MYB factors MYB20 and MYB14, MYB homeodomain protein, DNA binding protein, MYB-like transcription factors (Arabidopsis orthologs At3g16350 and At5g52660), and MYB-like protein were induced.

Supplementary data related to this article can be found in the online version, at http://dx.doi.org/10.1016/j.plantsci.2013.12.003.

\subsection{Secondary metabolism}

Transcriptomic changes in secondary metabolism affect organoleptic and aromatic properties of citrus fruits. No clear overall expression change was observed for genes involved in the non-mevalonate pathway. In symptomatic albedo, CLA1, DXR, and SPS2 were induced while CSB3, CLB6, and GGR were repressed. Three genes encoding geranylgeranyl transferase type II beta subunit were downregulated in both peel tissues. Phenylpropanoid biosynthesis genes also showed contrasting trends: some genes were repressed (methyltransferase 1, elicitor-activated gene 1, cinnamyl alcohol dehydrogenase, nicotinamidase, and transferase family proteins) while other genes encoding methyltransferases were upregulated. A few terpenoid genes were upregulated such as a camelliol synthase 1 and cycloartenol synthases. Tocophenol biosynthesis was altered in puffed fruits: two genes were induced (phytoene desaturation 1 and tocopherol methyltransferase 1) and others were repressed (homogentisate phytyltransferase 1 , albino or pale green mutant 1 gene, vitamin deficient 1 , and gamma-tocopherol methyltransferase).

\subsection{Mineral ion-related pathways}

Both creasing and puffing disorder were regulated by application of different mineral ions such as nitrogen, zinc, and magnesium [43]. This is consistent with GSEA data showing that transport pathways were affected by the disorder. Moreover, some genes associated with nitrogen metabolism (glutamate synthase, nitrate reductase, and glutamate dehydrogenase) and sulfate assimilation 
Table 3

Highly interactive proteins in puffing disorder.

\begin{tabular}{|c|c|c|c|c|}
\hline Citrus Affymetrix identifier & Arabidopsis ortholog & Albedo regulation & Number of PPI & Annotation \\
\hline Cit.30930.1.S1_S_at & AT4G26840 & Down & 494 & Heat shock protein 83 \\
\hline Cit.9298.1.S1_s_at & AT5G15200 & Down & 157 & Ubiquitin-like protein \\
\hline Cit.28354.1.S1_at & AT1G54290 & Up & 148 & Heat shock protein 70 \\
\hline Cit.1688.1.S1_s_at & AT2G37470 & Down & 141 & Farnesyldiphosphate synthase \\
\hline Cit.3349.1.S1_s_at & AT5G41480 & Up & 140 & Proteasome $26 \mathrm{~S}$ \\
\hline Cit.36150.1.S1_at & AT3G18660 & Up & 138 & GTP-binding protein (RABH1) \\
\hline Cit.26578.1.S1_at & AT5G54840 & Up & 121 & Rad51 \\
\hline Cit.1865.1.S1_s_at & AT5G15450 & Down & 112 & CDC2PNC \\
\hline Cit.11819.1.S1_S_at & АT3G16950 & Down & 103 & RNA pol II accessory factor \\
\hline Cit.8012.1.S1_s_at & AT5G10260 & Up & 101 & Histone $\mathrm{H} 2 \mathrm{~B}$ \\
\hline Cit.5947.1.S1_at & AT4G38630 & Up & 97 & Glycogenin-like starch initiation \\
\hline Cit.2004.1.S1_s_at & AT2G31260 & Up & 96 & Ubiquitin conjugating protein \\
\hline Cit.4642.1.S1_s_at & AT1G19120 & Down & 90 & Heat shock protein-like clpB \\
\hline Cit.11626.1.S1_s_at & AT5G52640 & Up & 89 & Small nuclear ribonucleoprotein \\
\hline Cit.29662.1.S1_s_at & AT3G01090 & Down & 89 & Binding protein, putative c-myc \\
\hline Cit.32365.1.S1_at & AT5G26340 & Up & 86 & Small nuclear ribonucleoprotein \\
\hline Cit.12003.1.S1_at & AT1G10210 & Down & 85 & Putative RNA binding protein \\
\hline Cit.14570.1.S1_s_at & AT5G23290 & Up & 81 & Microtubule-associated protein \\
\hline Cit.29702.1.S1_at & AT3G50670 & Up & 77 & Mitogen-activated protein kinase \\
\hline Cit.31720.1.S1_at & AT5G09590 & Up & 76 & Unknown protein \\
\hline Cit.18124.1.S1_at & AT4G21490 & Up & 74 & Factor splicing pre-mRNA \\
\hline Cit.5210.1.S1_at & AT5G20850 & Down & 74 & GTP-binding family protein \\
\hline Cit.11440.1.S1_S_at & AT3G18850 & Down & 74 & Phosphoglucomutase \\
\hline Cit.3923.1.S1_S_at & AT4G38130 & Down & 73 & Dihydrolipoamide dehydrogenase \\
\hline Cit.30646.1.S1_s_at & AT3G10070 & Up & 71 & Hexose transport protein \\
\hline Cit.29554.1.S1_S_at & AT2G30160 & Down & 71 & Protein U1 snRNP70 K \\
\hline Cit.16097.1.S1_at & АТ3G48750 & Up & 66 & KOW transcription factor \\
\hline Cit.24742.1.S1_at & AT2G02760 & Down & 66 & NADPH dehydrogenase \\
\hline Cit.24179.1.S1_at & AT1G19660 & Up & 65 & 1-acylglycerol-3-phosphate \\
\hline Cit.21317.1.S1_at & AT4G03430 & Up & 65 & Unknown protein \\
\hline Cit.32397.1.S1_at & AT5G67270 & Up & 61 & Unknown protein \\
\hline Cit.2363.1.S1_s_at & AT5G04600 & Up & 61 & Histone deacetylase \\
\hline Cit.9065.1.S1_S_at & AT4G17190 & Down & 61 & Ribosomal protein S940S \\
\hline Cit.8308.1.S1_s_at & AT4G08350 & Down & 61 & Putative translation factor \\
\hline Cit.18478.1.S1_at & АТ3G22590 & Down & 61 & Wound-responsive protein \\
\hline Cit.20717.1.S1_S_at & AT5G51820 & Up & 60 & Mitochondrial carrier protein \\
\hline Cit.7713.1.S1_at & AT2G43810 & Down & 60 & Folylpolyglutamate synthase \\
\hline
\end{tabular}

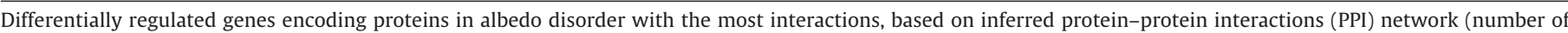
$\mathrm{PPI} \geq 60$ ).

(adenylylsulfate kinase and PAPS reductase) were significantly regulated by puffing disorder (Fig. 2).

\subsection{Protein-protein interaction network analysis}

A Citrus protein-protein interaction (PPI) network was inferred based on a protein interaction network database in Arabidopsis [25] with 29,793 predicted interactions. The file consisting of predicted pairwise interactions was loaded in Graphviz, producing a graph in which nodes represent proteins encoded by puffing disorder-regulated genes. Edges in the graph represent predicted pairwise interactions based on the predicted PPIs of Arabidopsis (Fig. S2). This "Citrus interactome" was used to identify key candidate genes encoding highly interactive proteins ("hubs"). Symptomatic fruit had differentially regulated hub proteins in both albedo and flavedo (Table 3). Of albedo proteins encoded by genes showing significant differential expression between puffed and control samples, heat shock proteins HSP82 and HSC70, farnesyl diphosphate synthase 2 (FPS2), rab GTPase homolog (RABH1), and ATRAD51 had the highest number of protein-protein interactions. However, it is also possible that genes with similar function to heat shock proteins could be induced in the absence of stresses [56]. Several hub proteins were regulated in both albedo and flavedo: HSP81-1, ubiquitin binding protein (AT-MCB1), cell division control 2 (CDC2), albino pale green 6 (APG6), and sugar transport protein 13 (MSS1).
Supplementary data related to this article can be found in the online version, at http://dx.doi.org/10.1016/j.plantsci.2013.12.003.

Transcriptional regulation of genes encoding highly interactive proteins could drastically affect the protein-protein interaction network. Genes identified in the present study must be validated by proteomics and enzyme assays to elucidate post-transcriptional regulatory mechanisms. We expect such analysis to show that heat shock (HSP82 and HSP70) and other highly interactive proteins play important roles in the appearance of puffing disorder symptoms. These hub genes belong to stress response pathways and to pathways involved in gene regulation (transcription factors), signaling, carbohydrates, transport, and hormones (brassinosteroid, ethylene, gibberellin, and jasmonate). Once validated, genes encoding many of these proteins may be appropriate targets for novel short-term therapeutic strategies.

\section{Conclusions}

Findings of this study suggest likely mechanisms underlying peel disorders in Citrus and other specialty crops. The data show differential expression of several key genes and altered concentrations of metabolites involved in glycolysis, the TCA cycle, and sucrose, starch, and raffinose metabolism. Many changes represent altered source-sink communications, with gibberellin playing a key role. These data may serve as a basis to develop methods for detecting early host response genes and metabolites (i.e. citric acid), with the potential for short-term therapeutic strategies 
based on the plant hormones gibberellins, cytokinins, and auxins, in addition to genetic (breeding or transformation) control strategies. Further validation would compare different hormones, doses, and environmental and physiological conditions of Citrus trees.

\section{Acknowledgements}

This work was supported by grants obtained from the Citrus Research Board of California. The authors wish to thank to Mary Lou Mendum for her editorial review of the manuscript.

\section{References}

[1] M. Agusti, V. Almela, M. Aznar, M. Elotmani, J. Pons, Satsuma mandarin fruit size increased by 2,4-DP, Hortscience 29 (1994) 279-281.

[2] J.M. Bove, Huanglongbing, A destructive, newly-emerging, century-old disease of citrus, J. Plant Pathol. 88 (2006) 7-37.

[3] M.T.Z.L. Lafuente, Postharvest physiological disorders in citrus fruit, Stewart Postharvest Rev. 2 (2006) 1-9

[4] D. Goudeau, S.L. Uratsu, K. Inoue, F.G. daSilva, A. Leslie, et al., Tuning the orchestra: selective gene regulation and orange fruit quality, Plant Sci. 174 (2008) 310-320.

[5] S.K. Kawase, K. Hirose, Use of growth regulators to control rind puffing in Satsuma mandarin fruit, in: Proceedings of International Society of Citriculture, 1981, pp. 237-239.

[6] Z. Wang, M. Gerstein, M. Snyder, RNA-Seq: a revolutionary tool for transcriptomics, Nat. Rev. Genet. 10 (2009) 57-63.

[7] M. Boccara, A. Sarazin, B. Billoud, V. Jolly, R. Martienssen, et al., New approaches for the analysis of Arabidopsis thaliana small RNAs, Biochimie 89 (2007) $1252-1256$.

[8] F. Martinelli, S.L. Uratsu, U. Albrecht, R.L. Reagan, M.L. Phu, et al., Transcriptome profiling of citrus fruit response to Huanglongbing disease, PLoS ONE 7 (2012) 5

[9] F. Martinelli, R.L. Reagan, S.L. Uratsu, M.L. Phu, U. Albrecht, et al., Gene regulatory networks elucidating Huanglongbing disease mechanisms, PLoS ONE (2013), http://dx.doi.org/10.1371/journal.pone.0074256.

[10] F. Martinelli, S.L. Uratsu, R.L. Reagan, Y. Chen, D. Tricoli, et al., Gene regulation in parthenocarpic tomato fruit, J. Exp. Bot. 60 (2009) 3873-3890.

[11] P. F.Blanco, N.M. Salinas, X. Cecchini, P. Jordana, M.E. Van Hummelen, L. Alvarez, Holuigue early genomic responses to salicylic acid in Arabidopsis, Plant Mol. Biol. 70 (2009) 79-102.

[12] F.M. Rizzini, C. Bonghi, L. Chkaiban, F. Martinelli, P. Tonutti, Effects of postharvest partial dehydration and prolonged treatments with ethylene on transcript profiling in skins of wine grape berries, Acta Hort. 877 (2010) 1099-1104.

[13] D.B. Kell, Metabolomics, machine learning and modelling in systems biology: towards an understanding of the language of cells, J. Biotechnol. 118 (2005) S5.

[14] F. Tosetti, F. Martinelli, D.K. Barupal, P. Tonutti, Metabolomics approach to studying minimally processed peach (Prunus persica) fruit, Acta Hort. (2012) 934.

[15] F. Martinelli, B. Basile, G. Morelli, R. d'Andria, P. Tonutti, Effects of irrigation on fruit ripening behavior and metabolic changes in olive, Sci. Hortic. 144 (2012) 201-207.

[16] F. Martinelli, D. Remorini, S. Saia, R. Massai, P. Tonutti, Metabolic profiling of ripe olive fruit in response to moderate water stress, Scientia Horticulturae 159 (2013) 52-58.

[17] D. Parker, M. Beckmann, H. Zubair, D.P. Enot, Z. Caracuel-Rios, et al., Metabolomic analysis reveals a common pattern of metabolic re-programming during invasion of three host plant species by Magnaporthe grisea, Plant J. 59 (2009) 723-737.

[18] C.Y. Wan, T.A. Wilkins, A modified hot borate method significantly enhances the yield of high quality RNA from cotton (Gossypium hirsutum L.), Anal. Biochem. 1 (1994) 7-12.

[19] R.A. Irizarry, B. Hobbs, F. Collin, Y.D. Beazer-Barclay, K.J. Antonellis, et al., Exploration, normalization, and summaries of high density oligonucleotide array probe level data, Biostatistics 4 (2003) 249-264.

[20] Y. Benjamini, Y. Hochberg, On the adaptive control of the false discovery fate in multiple testing with independent statistics, J. Educ. Behav. Stat. 25 (2000) 60-83.

[21] D.M. Rocke, Design and analysis of experiments with high throughput biological assay data, Semin. Cell Dev. Biol. 15 (2004) 703-713.

[22] O. Thimm, O. Blasing, Y. Gibon, A. Nagel, S. Meyer, et al., MAPMAN: a user-driven tool to display genomics data sets onto diagrams of metabolic pathways and other biological processes, Plant J. 37 (2004) 914-939.

[23] N. Goffard, T. Frickey, G. Weiller, PathExpress update: the enzyme neighbourhood method of associating gene-expression data with metabolic pathways, Nuclic Acids Res. 37 (2009) W335-W339.

[24] A. Conesa, S. Gotz, J.M. Garcia-Gomez, J. Terol, M. Talon, M. Robles, Blast2GO: a universal tool for annotation, visualization and analysis in functional genomics research, Bioinformatics 21 (2005) 3674-3676.
[25] J. Geisler-Lee, N. O’Toole, R. Ammar, N.J. Provart, A.H. Millar, M. Geisler, A predicted interactome for Arabidopsis, Plant Physiol. 145 (2007) 317-329.

[26] U. Albrecht, K.D. Bowman, Gene expression in Citrus sinensis (L.) Osbeck following infection with the bacterial pathogen Candidatus Liberibacter asiaticus causing Huanglongbing in Florida, Plant Sci. 175 (2008) 291-306.

[27] J.-S. Kim, U.S. Sagaram, J.K. Burns, J.-L. Li, N. Wang, Response of sweet orange (Citrus sinensis) to 'Candidatus Liberibacter asiaticus' infection: microscopy and microarray analyses, Phytopathology 99 (2009) 50-57.

[28] A.M. Dandekar, F. Martinelli, C.E. Davis, A. Bhushan, W. Zhao, O. Fiehn, K. Skogerson, G. Wohlgemuth, R. D’Souza, S. Roy, et al., Analysis of early host responses for asymptomatic disease detection and management of specialty crops, Crit. Rev. Immunol. 30 (2010) 277-289.

[29] R.A. Cernadas, L.R. Camillo, C.E. Benedetti, Transcriptional analysis of the sweet orange interaction with the citrus canker pathogens Xanthomonas axonopodis pv. citri and Xanthomonas axonopodis pv. aurantifolii, Mol. Plant Pathol. 9 (2008) 609-631.

[30] R. Pedreschi, E. Vanstreels, S. Carpentier, M. Hertog, J. Lammertyn, et al., Proteomic analysis of core breakdown disorder in conference pears (Pyrus communis L.), Proteomics 7 (2007) 2083-2099.

[31] A.R. Fernie, F. Carrari, L.J. Sweetlove, Respiratory metabolism: glycolysis, the TCA cycle and mitochondrial electron transport, Curr. Opin. Plant Biol. 7 (2004) 254-261.

[32] J.C. Jang, P. Leon, L. Zhou, J. Sheen, Hexokinase as a sugar sensor in higher plants, Plant Cell 9 (1997) 5-19.

[33] A. Sadka, D.B. Dewald, G.D. May, W.D. Park, J.E. Mullet, Phosphate modulates transcription of soybean VspB and other sugar inducible genes, Plant Cell 6 (1994) 737-749

[34] J.C. Jang, J. Sheen, Sugar sensing in higher plants, Plant Cell 6 (1994) 1665-1679.

[35] I. Neta-Sharir, O. Shoseyov, D. Weiss, Sugars enhance the expression of gibberellin-induced genes in developing petunia flowers, Physiol. Plantarum 109 (2000) 196-202.

[36] M. Rosecler, M. Rossetto, E. Purgatto, J. do Nascimento, F.M. Lajolo, et al., Effects of gibberellic acid on sucrose accumulation and sucrose biosynthesizing enzymes activity during banana ripening, Plant Growth Regul. 41 (2003) 207-214.

[37] M.L. Brenner, N. Cheikh, The role of hormones in photosynthate partitioning and seed filling, in: Plant Hormones and their Role in Plant Growth and Development, Springer, New York, 1987, pp. 474-493.

[38] H. Kende, J.A.D. Zeevaart, The five classical plant hormones, Plant Cell 9 (1997) 1197-1210.

[39] S. Yamaguchi, Y. Kamiya, Gibberellin biosynthesis: its regulation by endogenous and environmental signals, Plant Cell Physiol. 41 (2000) 251-257.

[40] L. Navarro, R. Bari, P. Achard, P. Lison, A. Nemri, et al., DELLAs control plant immune responses by modulating the balance and salicylic acid signaling, Curr. Biol. 18 (2008) 650-655.

[41] T. Kuraoka, Histological studies on the fruit development of the Satsuma orange with special reference to peel-puffing, Memoirs of Ehime University 8 (1962) 106.

[42] L. Pozo, W.J. Kender, J.K. Burns, U. Hartmond, A. Grant, Effects of gibberellic acid on ripening and rind puffing in 'Sunbust' mandarin, Proc. Fla. State Hort. Soc. 113 (2000) 102-105.

[43] S.P. Monselise, M. Weiser, N. Shafir, R. Goren, E.E. Goldschmidt, Creasing of orange peel - physiology and control, J. Hortic. Sci. 51 (1976) 341-351.

[44] J.P. Chun, F. Tamura, K. Tanabe, A. Itai, Physiological and chemical changes associated with watercore development induced by GA in Japanese pear 'Akibae' and 'Housui', J. Jpn. Soc. Hortic. Sci. 72 (2003) 378-384.

[45] F. Tamura, J.P. Chun, K. Tanabe, M. Morimoto, A. Itai, Effect of summer-pruning and gibberellin on the watercore development in Japanese pear 'Akibae' fruit, J. Jpn. Soc. Hortic. Sci. 72 (2003) 372-377.

[46] A. Garcialuis, M. Agusti, V. Almela, E. Romero, J.L. Guardiola, Effect of gibberellic acid on ripening and peel puffing in satsuma mandarin, Sci. Hortic. 27 (1985) 75-86.

[47] J.I.I. Lutinec, P. Vra, Gibberellin-induced changes in glycolysis and in lipid metabolism of wheat leaves, Biol. Plant. Acad. Sci. Bohemoslov. 5 (1963) 181-189.

[48] G. Ben-Nissan, D. Weiss, Developmental and hormonal regulation of a triosephosphate isomerase gene in petunia corollas, J. Plant Physiol. 147 (1995) 58-62.

[49] H. Konishi, H. Yamane, M. Maeshima, S. Komatsu, Characterization of fructose-bisphosphate aldolase regulated by gibberellin in roots of rice seedling, Plant Mol. Biol. 56 (2004) 839-848.

[50] C.-H. Lee, D.-H. Han, S.-B. Kim, Effects of GA-3 and fulmet (KT-30) on fruit set and quality in 'Kyoho' grapes, J. Korean Soc. Hortic. Sci. 37 (1996) 686-690.

[51] A. Heyl, T. Werner, T. Schmuelling, Cytokinin metabolism and signal transduction, in: P. Hedden, S.G. Thomas (Eds.), Plant Hormone Signaling, vol. 24, Blackwell Publishing Ltd., Oxford, 2006, pp. 93-123.

[52] X. Wang, B.M.V.S. Basnayake, H. Zhang, G. Li, W. Li, et al., The Arabidopsis ATAF1, a NAC transcription factor is a negative regulator of defense responses against necrotrophic fungal and bacterial pathogens, Mol. Plant Microbe Int. 22 (2009) 1227-1238.

[53] R.S. Cormack, T. Eulgem, P.J. Rushton, P. Kochner, K. Hahlbrock, et al., Leucine zipper-containing WRKY proteins widen the spectrum of immediate early elicitor-induced WRKY transcription factors in parsley, Biochim. Biophys. Acta Gene Struct. Exp. 157 (2002) 92-100. 
[54] S. Encinas-Villarejo, A.M. Maldonado, F. Amil-Ruiz, B. de los Santos, F. Romero, et al., Evidence for a positive regulatory role of strawberry (Fragaria $\times$ ananassa) Fa WRKY1 and Arabidopsis At WRKY75 proteins in resistance, J. Exp. Bot. 60 (2009) 3043-3065.

[55] S. Li, Q. Fu, W. Huang, D. Yu, Functional analysis of an Arabidopsis transcription factor WRKY25 in heat stress, Plant Cell Rep. 28 (2009) 683-693.
[56] L. Natali, T. Giordani, B. Lercari, P. Maestrini, R. Cozza, T. Pangaro, P. Vernieri, F Martinelli, A. Cavallini, Light induces expression of a dehydrin-encoding gene during seedling deetiolation in sunflower (Helianthus annuus L.), J. Plant Physiol. 164 (2007) 263-273. 